\title{
VALIDATION OF THE TRIPLE TIMED UP-AND-GO TEST IN LAMBERT-EATON MYASTHENIA
}

\author{
SHRUTI M. RAJA, MD ID, ${ }^{1}$ DONALD B. SANDERS, MD, ${ }^{1}$ VERN C. JUEL, MD, ${ }^{1}$ YADOLLAH HARATI, MD, ${ }^{2}$ A. GORDON SMITH, MD, ${ }^{3}$ \\ ROBERT PASCUZZI, MD, ${ }^{4}$ DAVID P. RICHMAN, MD, ${ }^{5}$ ANGIE WU, MS, ${ }^{6}$ KATHY L. ALEŠ, MD, ${ }^{7}$ LAURA R. JACOBUS, BS, ${ }^{7}$ \\ DAVID P. JACOBUS, MD, ${ }^{7}$ and JEFFREY T. GUPTILL, MD, ${ }^{1,8}$ ON BEHALF OF THE DAPPER STUDY TEAM ${ }^{\dagger}$ \\ ${ }^{1}$ Department of Neurology, Duke University Medical Center, Durham, North Carolina \\ ${ }^{2}$ Department of Neurology, Baylor College of Medicine, Houston, Texas \\ ${ }^{3}$ Department of Neurology, University of Utah School of Medicine, Salt Lake City, Utah \\ ${ }^{4}$ Department of Neurology, Indiana University School of Medicine, Indianapolis, Indiana \\ ${ }^{5}$ Department of Neurology, University of California Davis Medical Center, Sacramento, California \\ ${ }^{6}$ Center for Predictive Modeling, Duke Clinical Research Institute, Durham, North Carolina \\ ${ }^{7}$ Jacobus Pharmaceutical Company, Princeton, New Jersey \\ ${ }^{8}$ Neurosciences Medicine, Duke Clinical Research Institute, Durham, North Carolina \\ Accepted 29 June 2019
}

ABSTRACT: Introduction: There are no validated, practical, and quantitative measures of disease severity in Lambert-Eaton myasthenia (LEM). Methods: Data from the Effectiveness of 3,4-Diaminopyridine in Lambert-Eaton Myasthenic Syndrome (DAPPER) trial were analyzed to assess triple timed up-and-go (3TUG) reproducibility and relationships between 3TUG times and other measures of LEM severity. Results: The coverage probability technique showed $\geq 0.90$ probability for an acceptable 3TUG difference of $\leq 0.2$, indicating that it is reproducible in LEM patients. The correlation between 3TUG times and lower extremity function scores was significant in subjects who continued and in those who were withdrawn from 3,4-diaminopyridine free base. Worsening patientreported Weakness Self-Assessment Scale and Investigator Assessment of Treatment Effect scores corresponded with prolongation of 3TUG times. Discussion: The 3TUG is reproducible, demonstrates construct validity for assessment of lower extremity function in LEM patients, and correlates with changes in patient and physician assessments. These findings, along with prior

Additional supporting information may be found in the online version of this article.

This is an open access article under the terms of the Creative Commons Attribution-NonCommercial-NoDerivs License, which permits use and distribution in any medium, provided the original work is properly cited, the use is non-commercial and no modifications or adaptations are made.

Abbreviations: 3TUG, triple timed up-and-go test; CMAP, compound muscle action potential; CP, coverage probability; DAP, 3,4-diaminopyridine free base; DAPPER, Effectiveness of 3,4-Diaminopyridine in Lambert-Eaton Myasthenic Syndrome trial; LEFS, Lower Extremity Function Scale; LEM, Lambert-Eaton myasthenia; LEM-ADL, Lambert-Eaton Myasthenia Activities of Daily Living; MCID, minimal clinically important difference; $Q M G$, quantitative myasthenia gravis; W-SAS, Weakness Self-Assessment Scale

Key words: Lambert-Eaton myasthenia, Lambert-Eaton myasthenic syndrome, Lambert-Eaton syndrome, outcome measures, timed up-and-go test, validation

Present address

A. Gordon Smith, Virginia Commonwealth University, Richmond, VA

Funding information American Association of Neuromuscular and Electrodiagnostic Medicine; Shire; National Institute of Neurological Disorders and Stroke of the National Institutes of Health, Grant Award Number: K23NS085049 (to J.T.G.)

'Refer to Appendix for a listing of the Effectiveness of 3,4-Diaminopyridine in Lambert-Eaton Myasthenic Syndrome (DAPPER) study investigators.

This article was first presented at the scientific session of the Myasthenia Gravis Foundation of America annual meeting of the American Association of Neuromuscular and Electrodiagnostic Medicine, October 2018, Washington, DC

Correspondence

Shruti M. Raja, Duke University Medical Center, Durham, NC. Email: shruti. raja@duke.edu

(C) 2019 Wiley Periodicals, Inc

Published online 3 July 2019 in Wiley Online Library (wileyonlinelibrary.

com). DOI 10.1002/mus.26625 reliability studies, indicate $3 T$ TUG is a valid measure of disease severity in LEM.

Muscle Nerve 60:292-298, 2019

\section{INTRODUCTION}

Lambert-Eaton myasthenia (LEM) is a rare and debilitating disorder of neuromuscular transmission caused by autoantibodies to the P/Q-type voltagegated calcium channels (VGCCs) of the presynaptic neuromuscular junction. ${ }^{1}$ Patients typically present with weakness in the shoulder, hip, and thigh muscles $^{2}$ and autonomic dysfunction. ${ }^{3}$ Dysphagia and respiratory failure ${ }^{4}$ can be prominent in some patients. The decline in lower extremity strength leads to impaired mobility ${ }^{5}$ and poor quality of life. ${ }^{6}$

Several single-center studies ${ }^{7-11}$ have demonstrated improvement in LEM-associated weakness after administration of the potassium channel antagonist ${ }^{12-14}$ 3,4-diaminopyridine free base (DAP). Previous treatment trials of DAP in LEM have used change in strength of selected muscles,${ }^{11}$ the quantitative myasthenia gravis (QMG) score, ${ }^{12,13}$ or myometry $^{8,11}$ as primary outcome measures, with compound muscle action potential (CMAP) amplitude $^{8,11-13}$ as a secondary outcome measure in some trials. Autonomic function testing, patients' subjective symptoms, and physicians' disease classification have also been used as measures of disease severity in LEM trials. None of these measures have been validated in LEM, and others, including the QMG, do not measure important causes of LEMrelated disability, such as proximal lower extremity weakness and diminished mobility. Electrodiagnostic studies are time-consuming and the availability of some techniques is limited to specialized centers, making them impractical as routine clinical measures of disease severity.

An optimal outcome measure for LEM should fulfill the basic requirements proposed by Hobart et $\mathrm{al}^{15}$ and that it be clinically practical and 
scientifically sound on the basis of validity, reliability, and responsiveness.

The timed up-and-go (TUG) test ${ }^{16}$ is a simple objective measure of mobility that has been validated primarily in geriatric populations with parkinsonism ${ }^{17,18}$ and impaired cognition. ${ }^{19,20}$ It requires the patient to rise up from the seat of a straight-backed armchair, walk 3 meters at a normal pace, turn around, walk back to the chair, turn, and sit back down. A variation of this test, the triple timed up-and-go (3TUG) test, requires 3 repetitions (laps), and assesses the lower extremity weakness and fatigue or facilitation characteristic of LEM. The 3TUG has been demonstrated to have excellent test-retest reproducibility and interrater reliability in patients with non-LEM neuromuscular disorders, but has not been fully validated in LEM.

This study was designed to further validate the 3TUG in LEM patients by confirming test-retest reproducibility and interrater reliability, establishing construct validity through correlation with other measures of LEM-specific disability, and assessing its responsiveness to patient- and provider-reported measures of disease severity.

\section{METHODS}

This study was a secondary analysis of data from the Effectiveness of 3,4-Diaminopyridine in Lambert-Eaton Myasthenic Syndrome (DAPPER) clinical trial, a double-blind, placebo-controlled withdrawal study of DAP in patients with LEM (NCT01511978). ${ }^{22}$ The trial consisted of 4 stages (refer to Figure S1 in the Supplementary Material online): Acclimation (0.5 day); Baseline (2 days); Withdrawal (up to 3.5 days); and DAP Reinstitution (0.5-2 days). Data from participants who completed at least the acclimation and baseline observation stages were included in this analysis. Participants were randomized to receive DAP or placebo as per home schedules. Time-points for non-3TUG measures (see Figure S1 online) were determined based on when they were performed before randomization or during withdrawal, and were matched with the closest 3TUG time. Lower Extremity Function Scale (LEFS) scores were obtained during Acclimation (day 0) and again at the end of Withdrawal or time of early advancement; all other baseline measurements were obtained during Baseline (day 2).

Oversight. The institutional review board of the Duke University Health System exempted this study (Pro00083458) from review.

Outcome measures. The 3TUG was performed before and after the first DAP doses of the afternoon and evening with 4 test times daily during the Acclimation stage and 6 times daily (including before and after first morning dose) during the Baseline and Withdrawal stages.

CMAPs were measured in the muscle determined to be most responsive to DAP during the Acclimation stage and were reviewed for quality by a blinded observer (see Table S1 online). CMAP amplitude was measured before and after the first doses of the morning and afternoon during the Baseline and Withdrawal stages.

The LEFS (see Figure S2 online) is a 20-item patientreported outcome measure commonly used to assess mobility in patients with orthopedic conditions. ${ }^{23}$ The best possible score is 80 , and a change of \pm 9 points indicates a clinically meaningful change in functional ability. Although this tool has not been used in patients with LEM, the predominant lower extremity and hip-girdle weakness in LEM is consistent with symptoms experienced by patients with musculoskeletal problems.

The Weakness Self-Assessment Scale (W-SAS) is a secondary efficacy measure created by the DAPPER trial sponsor (see Figure S3 online). It features 7 categories with numerical values that allow a participant to rank weakness along a continuum from "Much much weaker" (-3) to "Much much stronger" (+3). The W-SAS was performed 2 hours after the first DAP doses of the afternoon and evening with 2 test times daily during Baseline and 3 times daily (including first morning dose) during Withdrawal.

The Investigator Assessment of Treatment Effect is a 5-item categorical scale created by the DAPPER trial sponsor (see Figure S4 online). It was performed by a study-blind investigator to assess overall disease severity at the conclusion of withdrawal or at the time of early advancement. Participants were assessed along a continuum from "Much worse than during baseline" (0) to "Much improved from baseline" (4).

Statistical analysis. Reproducibility and reliability analyses were performed using data from participants who completed the Acclimation and Baseline stages. The other analyses included only randomized participants. Because the goal of this validation study was to evaluate 3TUG performance over time in participants who are clinically stable (continuous DAP) and clinically changing (controlled DAP withdrawal), between-group comparisons of outcome measures for those who continued DAP and those who were withdrawn from DAP were not performed; these have been reported previously. ${ }^{22}$ All analyses were performed using a percentage change from Baseline rather than the absolute values at the prespecified time-points. Data were analyzed using SAS version 9.4 (SAS Institute, Cary, North Carolina).

Test-retest reproducibility and interrater reliability of 3TUG. Time-matched 3TUG times recorded by the same onsite observer for the same participant on 2 consecutive days (Figure 1) were analyzed to assess test-retest reproducibility. Participants continued their home doses of DAP during these stages, and only data from participants with values for both time-points were included. Agreement between 2 observations of the same 3TUG was determined by comparing 3TUG times recorded by an onsite observer and a remote observer who viewed videotaped 3TUGs. Only participants with observations by 2 different observers were included $(n=46)$. The coverage probability (CP) method was used to assess agreement between paired observations. ${ }^{24}$ A CP value is the probability that the ratio between paired observations falls within a preestablished range: it is calculated by dividing the number of observed ratios within the acceptable range by the total number of comparisons. For this study, it was established a priori that agreement would be demonstrated by a $\mathrm{CP} \geq 0.90$. Point estimates and a 95\% CIs for the CP were calculated for an acceptable difference of $\leq 20 \%$. A sensitivity analysis for an acceptable difference of $\leq 10 \%$ was also performed. BlandAltman plots were constructed.

3TUG times vs CMAP amplitudes. The association between change in 3TUG time recorded by an onsite observer and change in time-matched CMAP by treatment group was assessed using data from subjects with both non-missing postdose 3TUG times and CMAP amplitudes for the first afternoon dose at baseline and 


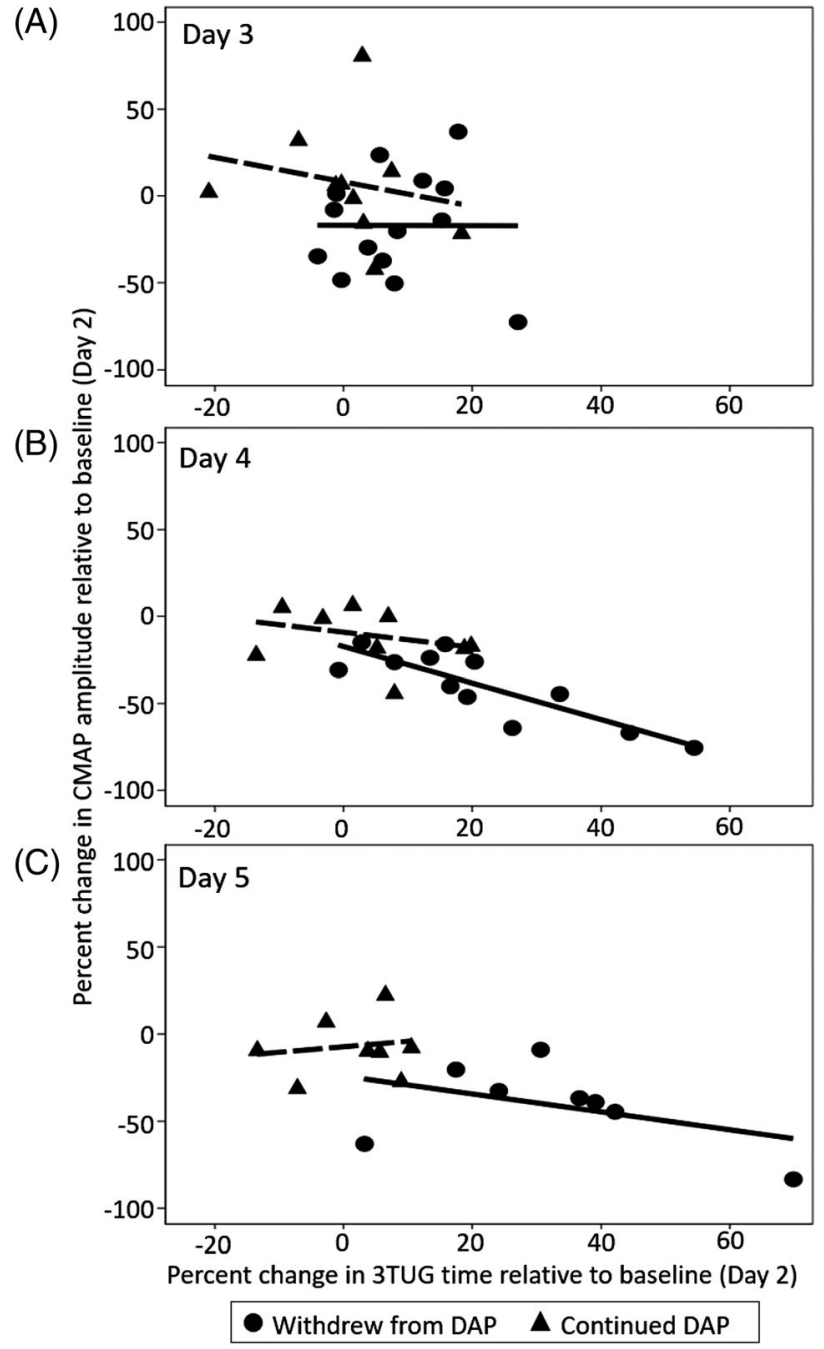

FIGURE 1. Percent change in CMAP amplitude vs percent change in 3TUG time during Withdrawal (stage II). CMAP amplitude and 3TUG time are standardized to Baseline (day 2). Lines of best fit show an association between percent change in CMAP amplitude and percent change in 3TUG time as DAP is progressively withdrawn on days $3(\mathrm{~A})$ and $4(\mathrm{~B})$. The association is not apparent on day 5 (C). 3TUG, triple timed up-and-go test; CMAP, compound muscle action potential; DAP, 3,4-diaminopyridine free base

during each day of the withdrawal phase. Percent change in 3TUG time and CMAP amplitude were calculated using the following formula:

$[($ Postdose withdrawal-Postdose baseline $) /($ Postdose baseline $)] \times 100$

Descriptive statistics for the mean absolute and percentage change in 3TUG time and CMAP amplitude were calculated. A multivariable linear regression was fit to the data by randomization group using the prespecified model:

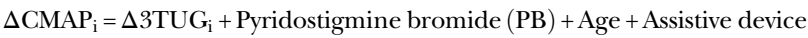

Only statistically significant $(P<.05)$ adjustment variables were reported in the final model due to risk of overfitting with the small sample size. A simplified univariate linear regression was reported as: $\Delta \mathrm{CMAP}_{\mathrm{i}}=\Delta 3 \mathrm{TUG}_{\mathrm{i}}$.

3TUG times vs LEFS scores. The association between change in 3TUG time recorded by an onsite observer and change in LEFS scores by treatment group was assessed using data from participants with time-matched postdose 3TUG times and LEFS scores from baseline and at the end of Withdrawal. Percent change in 3TUG time was calculated using the following formula:

$[($ Withdrawal value - Baseline value $) /($ Baseline value $)] \times 100$

Raw change in the LEFS score was calculated by subtracting the Baseline value from the Withdrawal value.

Descriptive statistics were calculated for the change in 3TUG time and LEFS score at each time-point. Spearman correlations between the time-matched 3TUG time and LEFS scores and percent change in 3TUG time and raw change in LEFS scores were determined.

3TUG times vs W-SAS. The association between change in 3TUG time and change in W-SAS score was assessed by comparing the last available time-matched 3TUG time and W-SAS score in the Withdrawal stage (days 4 or 5) and the last available time-matched W-SAS and 3TUG time during Baseline stage (day 2). Descriptive statistics were calculated for the percent change in 3TUG time and change in numerical W-SAS score for each randomization group.

3TUG times vs Investigator Assessment of Treatment Effect. The association between change in 3TUG time recorded by an onsite observer and overall Investigator Assessment was determined by comparing the change in 3TUG time to the Investigator Assessment at the end of withdrawal. Only participants with documented values for both 3TUG and the investigator assessment at the end of Withdrawal were included in the analysis.

\section{RESULTS}

Test-retest reproducibility of 3TUG. Forty-six pairs of observations recorded by onsite observers were analyzed (Table 1). The CP for agreement in timematched observations on consecutive days is 0.93 (95\% CI, 0.82-0.99) for an acceptable range of $\leq 20 \%$, and 0.67 (95\% CI, 0.54-0.81) for an acceptable range of $\leq 10 \%$ (see Figure $\mathrm{S} 5$ online).

Table 1. Reproducibility and reliability of 3TUG

\begin{tabular}{|c|c|c|c|c|}
\hline \multirow[b]{2}{*}{ Parameter (s) } & \multicolumn{2}{|c|}{$\begin{array}{l}\text { Test-retest } \\
\text { reproducibility* }\end{array}$} & \multicolumn{2}{|c|}{ Interrater reliability ${ }^{\dagger}$} \\
\hline & Test 1 & Test 2 & Observer 1 & Observer 2 \\
\hline Mean 3TUG & 10.2 & 10.0 & 10.0 & 10.0 \\
\hline SD & 2.9 & 2.8 & 2.8 & 2.9 \\
\hline Mean difference & \multicolumn{2}{|c|}{-0.2} & \multicolumn{2}{|c|}{0.0} \\
\hline Minimum & \multicolumn{2}{|c|}{-2.5} & \multicolumn{2}{|c|}{-0.5} \\
\hline Maximum & \multicolumn{2}{|c|}{4.3} & \multicolumn{2}{|c|}{0.8} \\
\hline SD & \multicolumn{2}{|c|}{1.2} & \multicolumn{2}{|c|}{0.2} \\
\hline
\end{tabular}

Abbreviation: 3TUG, triple timed up-and-go test.

*Postdose following the first afternoon dose on day 0 and day 1 .

†Postdose following the first afternoon dose on day 1. Forty-six paired observations are represented in each analysis. 
Interrater reliability of 3TUG. Forty-six pairs of observations from Baseline were analyzed (Table 1). The CP for agreement between unblinded and blinded observers for the same 3TUG test was 1.00 (95\% CI, $0.92-1.00)$ for an acceptable range of $\leq 20 \%$, and 1.00 (95\% CI, 0.92-1.00) for an acceptable range of $\leq 10 \%$ (see Figure S6 online).

3TUG time vs CMAP amplitude. Between baseline and the last postdose CMAP of the Withdrawal period (study day 5 or early advancement), 3TUG times increased by a mean of $1.5 \%(95 \% \mathrm{CI},-0.4$ to $0.6)$ in those who continued DAP $(n=8)$ and by a mean of $32.9 \%$ (95\% CI, 16.4-49.3) in those who were withdrawn from DAP $(n=8)$ (see Table S2 online). In this same time period, the CMAP decreased by a mean of $-6.9 \%$ (95\% CI, -21.2 to 7.5) in those who continued DAP and by a mean of $-40.9(-3.4 \mathrm{mV}$; $95 \% \mathrm{CI},-60.5$ to -21.3$)$ in those who were withdrawn from DAP (see Table S3 online). Scatterplots with a line of best fit suggest a trend of decreasing CMAPs with increasing 3TUG times (Figure 1). Linear regression revealed a significant $(P<.01)$ association between 3TUG time and CMAP amplitude during the second day of the Withdrawal stage (study day 4) in those who were withdrawn from DAP; in these subjects, an increase of $1 \%$ in 3TUG time was associated with a $-1.05 \%$ (95\% CI, -1.52 to -0.57$)$ change in CMAP amplitude (Table 2). This association was not significant at Baseline or at the end of Withdrawal (study day 5) in either group.

3TUG times vs LeFs scores. Between baseline and the LEFS at the end of the withdrawal stage, the 3TUG time decreased by a mean of $1.9 \%(0.1$ second) in the group that continued DAP $(n=13)$ and increased by $126.3 \%$ (14.4 seconds) in the group that was withdrawn from DAP $(n=15)$. LEFS scores decreased by a mean of 2 points in the continued treatment group and decreased by 24 points in the group that was withdrawn from DAP. Spearman correlation showed a strong negative correlation between the 3TUG time and the total LEFS score before reinstitution of DAP in the continued DAP group $(r=-0.64, P=.02)$ and in those who were withdrawn from DAP $(r=-0.64, P=.01)$.

3TUG times vs W-sAs. Between Baseline and the end of the Withdrawal stage, the 3TUG time increased by a mean of $2.1 \%$ ( 0.2 second $)$ in the group that continued DAP $(\mathrm{n}=13)$ and by $81.5 \%$ (9.3 seconds) in the group that was withdrawn from DAP $(n=16)$. Among those withdrawn from DAP, an increase in 3TUG time was associated with a greater decline in W-SAS score (Figure 2A). Worsening of at least $3 \mathrm{~W}$-SAS points corresponded with a mean prolongation of $\geq 73.6 \%$ of 3 TUG time. Across both treatment groups, 3 of $11(27.3 \%)$ participants who reported feeling "Much, much weaker" were unable to perform the $3 \mathrm{TUG}$.

3TUG times vs Investigator Assessment of Treatment Effect. Blinded investigators assessed participants at baseline and at the end of the withdrawal period. Participants who were graded "Much worse" than at baseline had a mean increase of $\geq 94.3 \%$ in their 3TUG time and all were withdrawn from DAP $(\mathrm{n}=18)$; no participants who continued DAP $(\mathrm{n}=13)$ were rated as "Much worse" (Figure 2B). Across both treatment groups, 3TUG times deteriorated by $\geq 30 \%$ in $81.3 \%$ (13 of 16) of participants who were rated as "Somewhat worse" (4 of 16) or "Much worse" (12 of 16). Among those rated as "Much worse," $91.7 \%$ (11 of 12) had a $\geq 30 \%$ deterioration in 3TUG. A total of 3 participants who were rated as "Much worse" were not able to perform the 3TUG. No participants were reported as "Somewhat better" or "Much better."

\section{DISCUSSION}

The results of this study demonstrate that the 3TUG has both excellent reproducibility and interrater reliability in a population of LEM patients on stable

Table 2. Association between change from baseline postdose CMAP amplitude and change from baseline postdose 3TUG*

\begin{tabular}{lcccc} 
& \multicolumn{2}{l}{ Continuous DAP } & & Withdrew from DAP \\
\cline { 2 - 3 } & Effect estimate $(95 \% \mathrm{Cl})$ & & Effect estimate $(95 \%$ Cl) & \\
\hline Day $3^{\dagger}$ & $-0.70(-3.33$ to 1.92$)$ & 0.55 & $-0.01(-2.20$ to 2.18$)$ & \\
Day $4^{\ddagger}$ & $-0.43(-1.65$ to 0.79$)$ & 0.43 & $-1.05(-1.52$ to -0.57$)$ & 0.99 \\
Day $5^{\S}$ & $0.32(-1.69$ to 2.32$)$ & 0.71 & $-0.52(-1.59$ to 0.56$)$ & 0.01 \\
\hline
\end{tabular}

Abbreviations: 3TUG, triple timed up-and-go test; CMAP, compound muscle action potential; DAP, 3,4-diaminopyridine free base.

Only 3TUG-CMAP pairs with CMAPs considered acceptable by the study-blind reviewer were included in the analysis for each day.

$* \triangle \mathrm{CMAP}_{\mathrm{i}}=\Delta 3 \mathrm{TUG}_{\mathrm{i}}$.

Continued on DAP, $n=10$; withdrawn from DAP, $n=14$.

"Continued on DAP, $n=9$; withdrawn from DAP, $n=12$.

${ }^{\S}$ Continued on DAP, $n=8$; withdrawn from DAP, $n=8$.

Validation of 3TUG 

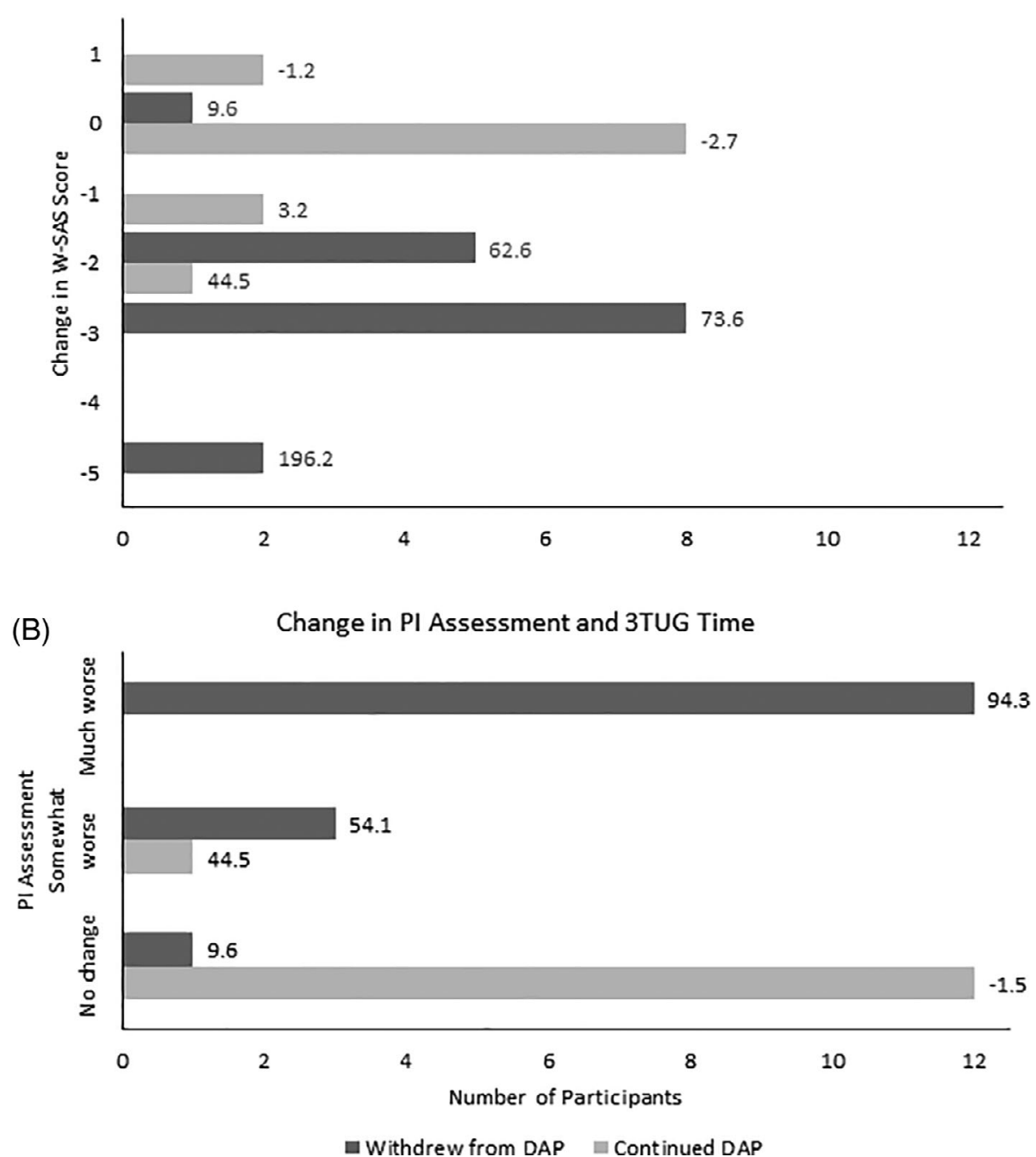

FIGURE 2. (A) Number of responders by change in W-SAS score. The numerical value represents the mean percent change in $3 T$ TUG time for each W-SAS score. (B) Number of responders by change in investigator assessment of overall treatment effect. The numerical value represents the mean percent change in 3TUG time for each Investigator Assessment category. 3TUG, triple timed up-and-go test; DAP, 3,4-diaminopyridine free base; PI, principal investigator; W-SAS, Weakness Self-Assessment Scale

therapy and has content and face validity for the assessment of lower extremity dysfunction. The DAPPER trial demonstrated that the W-SAS accurately captures patient perceptions of weakness, ${ }^{22}$ and analysis of the 3TUG has revealed that prolongation of 3TUG times corresponds with declines in W-SAS scores. In addition, deteriorations in 3TUG times are congruent with worsening, as assessed by blinded investigators. Together, these results indicate that the 3TUG is responsive to patient- and clinician-reported changes in disease severity. This offers an advantage over electrophysiological measures because it can be performed without special equipment and technical training.

Although there is no "gold standard" of LEM severity for comparison, the 3TUG can be compared with other measures of lower extremity function and mobility, such as the validated LEFS, with which it correlated well at the end of the withdrawal period, both in participants who continued DAP and in those who were withdrawn from DAP. Earlier work ${ }^{23}$ has established the clinically meaningful change in LEFS score to be \pm 9 points, consistent with observations in the DAPPER LEM participants who were withdrawn from DAP (mean, -22; range, -33 to -8 ), providing content validity of the 3TUG as an assessment of lower extremity function.

The present study has several limitations related to the comparator instruments and small sample sizes. The effect estimate of CMAP amplitude and 3TUG times was significant only in the middle of the withdrawal period (study day 4) for those who were withdrawn from DAP. This was unexpected because earlier reports demonstrated increases in CMAP amplitude after DAP administration. ${ }^{10,11,14,25}$ Several factors may have played a role in these inconsistent results, among 
them the small number of participants, particularly at the end of withdrawal, due to rescue, and differences in the CMAP technique used at the different study sites. ${ }^{26-28}$ Indeed, CMAPs from only 10 of $12(83 \%)$ of participants who continued DAP met the predetermined criteria for acceptability (see Table S3 online) on the first day of withdrawal (study day 3) and the number of acceptable CMAP studies was lower at each subsequent assessment. This pattern was also observed in the group withdrawn from DAP: only 14 of 18 (78\%) participants had acceptable CMAP studies on study day 3, and the number of acceptable CMAP studies declined at each subsequent assessment. These findings highlight the need for careful attention to the technical aspects of performing electrodiagnostic measures, and the potential limitations of using CMAP amplitudes in multicenter clinical trials unless rigorous training is employed. These concerns are shared with regard to other electrodiagnostic measures, as recently highlighted in an editorial on the use of motor unit number index (MUNIX) in clinical trials. ${ }^{29}$ An alternative methodology using longitudinal analysis strategies, such as mixed-effects modeling, in an adequately powered study, may provide a more accurate assessment of the relationship between pre- and postdose 3TUG times and CMAP amplitudes, and could also assess clinically meaningful covariates such as age, use of pyridostigmine, and assistive devices.

Although the CP for agreement in the test-retest reproducibility of the 3TUG was 0.93 for an acceptable range of $\leq 20 \%$, the CP for an acceptable range of $\leq 10 \%$ was 0.67 . This suggests the possibility of skew and dispersion in the data. The original DAPPER analysis did not report data from day 0 because of concerns that fatigue due to travel could have an unpredictable effect on the data.

This study has expanded upon the current knowledge of outcome measures in LEM and demonstrates that the 3TUG is reproducible in LEM patients, consistently scored by observers, and has both content and construct validity for LEMassociated disability. The 3TUG is a practical, validated outcome measure for clinical assessment of LEM patients that is suitable for use in the clinic, as well as in clinical trials.

\section{ACKNOWLEDGMENTS}

The authors thank Kevin Weinfurt, PhD, and Carl Pieper, DPH, for providing critical appraisal of the manuscript. The data for this study were obtained by the Jacobus Pharmaceutical Company.

\section{CONFLICT OF INTERESTS}

D.B.S. is or has been, during the past 12 months, a consultant to Accordant Health Services, Alexion, BD, Deallus, Jacobus Pharmaceutical Company, GSK, TerumoBCT, MorphoSys, and UCB. K.A. is medical director, L.R.J. is vice-president, and D.P.J. is president of the Jacobus Pharmaceutical Company. The remaining authors have no conflicts of interest to disclose. Full disclosures are available at http://www.dcri.duke.edu/ research/coi.

\section{AUTHOR DISCLOSURES}

S.M.R. is supported by an Autoimmune Neuromuscular Fellowship sponsored by the American Association of Neuromuscular and Electrodiagnostic Medicine (AANEM) and Shire. D.B.S. is or has been during the past 12 months, a consultant to Accordant Health Services, Alexion, BD, Deallus, Jacobus Pharmaceutical Co., GSK, TerumoBCT, MorphoSys and UCB. K.A. is medical director, Jacobus Pharmaceutical Company, Inc. L.R.J. is vice president, Jacobus Pharmaceutical Company, Inc. D.P.J. is president, Jacobus Pharmaceutical Company, Inc.

\section{ETHICAL PUBLICATION STATEMENT}

We confirm that we have read the Journal's position on issues involved in ethical publication and affirm that this report is consistent with those guidelines.

\section{REFERENCES}

1. Motomura M, Lang B, Johnston I, Palace J, Vincent A, Newsom-Davis J. Incidence of serum anti-P/Q-type and anti-N-type calcium channel autoantibodies in the Lambert-Eaton myasthenic syndrome. J Neurol Sci. 1997;147:35-42.

2. Meriggioli MN, Howard JF, Harper CM. Neuromuscular Junction Disorders: Diagnosis and Treatment. New York, NY: Marcel Dekker; 2004. p 166-190.

3. Waterman SA. Autonomic dysfunction in Lambert-Eaton myasthenic syndrome. Clin Auton Res. 2001;11:145-154.

4. Smith AG, Wald J. Acute ventilatory failure in Lambert-Eaton myasthenic syndrome and its response to 3,4-diaminopyridine. Neurology. 1996;46:1143-1145.

5. Wirtz PW, Wintzen AR, Verschuuren IJ. Lambert-Eaton myasthenic syndrome has a more progressive course in patients with lung cancer. Muscle Nerve. 2005;32:226-229.

6. Mantegazza R, Meisel A, Sieb JP, Le Masson G, Desnuelle C, Essing M. The European LEMS Registry: Baseline demographics and treatment approaches. Neurol Ther. 2015;4:105-124.

7. Lundh H, Nilsson $\mathrm{O}$, Rosen I. Treatment of Lambert-Eaton syndrome: 3,4-Diaminopyridine and pyridostigmine. Neurology. 1984;34:1324-1330.

8. McEvoy KM, Windebank AJ, Daube JR, Low PA. 3,4-Diaminopyridine in the treatment of Lambert-Eaton myasthenic syndrome. N Engl J Med. 1989;321:1567-1571.

9. Sanders DB, Howard JF Jr, Massey JM. 3,4-Diaminopyridine in Lambert-Eaton myasthenic syndrome and myasthenia gravis. Ann NY Acad Sci. 1993;681:588-590.

10. Oh SJ, Claussen GG, Hatanaka Y, Morgan MB. 3,4-Diaminopyridine is more effective than placebo in a randomized, double-blind, cross-over drug study in LEMS. Muscle Nerve. 2009;40:795-800.

11. Wirtz PW, Verschuuren JJ, van Dijk JG, de Kam ML, Schoemaker RC, van Hasselt JG, et al. Efficacy of 3,4-diaminopyridine and pyridostigmine in the treatment of Lambert-Eaton myasthenic syndrome: A randomized, double-blind, placebo-controlled, crossover study. Clin Pharmacol Ther. 2009;86:44-48.

12. Kirsch GE, Narahashi T. 3,4-Diaminopyridine. A potent new potassium channel blocker. Biophys J. 1978;22:507-512.

13. Molgo J, Lundh H, Thesleff S. Potency of 3,4-diaminopyridine and 4-aminopyridine on mammalian neuromuscular transmission and the effect of pH changes. Eur J Pharmacol. 1980;61:25-34.

14. Sanders DB, Massey JM, Sanders LL, Edwards LJ. A randomized trial of 3,4-diaminopyridine in Lambert-Eaton myasthenic syndrome. Neurology. 2000;54:603.

15. Hobart JC, Lamping DL, Thompson AJ. Evaluating neurological outcome measures: The bare essentials. J Neurol Neurosurg Psychiatry. 1996;60:127-130.

16. Podsiadlo D, Richardson S. The timed "Up \& Go": A test of basic functional mobility for frail elderly persons. J Am Geriatr Soc. 1991;39:142-148.

17. Morris S, Morris ME, Iansek R. Reliability of measurements obtained with the Timed "Up \& Go" test in people with Parkinson disease. Phys Ther. 2001;81:810-818. 
18. Huang SL, Hsieh CL, Wu RM, Tai CH, Lin CH, Lu WS. Minimal detectable change of the timed "up \& go" test and the dynamic gait index in people with Parkinson disease. Phys Ther. 2011;91: 114-121.

19. Ries JD, Echternach JL, Nof L, Gagnon Blodgett M. Test-retest reliability and minimal detectable change scores for the timed "up \& go" test, the six-minute walk test, and gait speed in people with Alzheimer disease. Phys Ther. 2009;89:569-579.

20. Nordin E, Rosendahl E, Lundin-Olsson L. Timed "Up \& Go" test: Reliability in older people dependent in activities of daily living-focus on cognitive state. Phys Ther. 2006;86:646-655.

21. Sanders DB, Guptill JT, Ales KL, Hobson-Webb LD, Jacobus DP, Mahmood R, et al. Reliability of the triple-timed up-and-go test. Muscle Nerve. 2018;57:136-139.

22. Sanders DB, Juel VC, Harati Y, Smith AG, Peltier AC, Marburger T, et al. 3,4-diaminopyridine base effectively treats the weakness of Lambert-Eaton myasthenia. Muscle Nerve 2018;57:561-568.

23. Binkley JM, Stratford PW, Lott SA, Riddle DL. The Lower Extremity Functional Scale (LEFS): Scale development, measurement properties, and clinical application. North American Orthopaedic Rehabilitation Research Network. Phys Ther. 1999;79:371-383.
24. Barnhart HX, Yow E, Crowley AL, Daubert MA, Rabineau D, Bigelow R, et al. Choice of agreement indices for assessing and improving measurement reproducibility in a core laboratory setting. Stat Methods Med Res. 2016;25:2939-2958.

25. Maddison P, Newsom-Davis J, Mills KR. Effect of 3,4-diaminopyridine on the time course of decay of compound muscle action potential augmentation in the Lambert-Eaton myasthenic syndrome. Muscle Nerve. 1998;21:1196-1198.

26. Tim RW, Massey JM, Sanders DB. Lambert-Eaton myasthenic syndrome: Electrodiagnostic findings and response to treatment. Neurology. 2000; 54:2176-2178

27. Chiou-Tan FY, Gilchrist JM. Repetitive nerve stimulation and single-fiber electromyography in the evaluation of patients with suspected myasthenia gravis or Lambert-Eaton myasthenic syndrome: Review of recent literature. Muscle Nerve. 2015;52:455-462.

28. AAEM Quality Assurance Committee of the American Association of Electrodiagnostic Medicine. Practice parameter for repetitive nerve stimulation and single fiber EMG evaluation of adults with suspected myasthenia gravis or Lambert-Eaton myasthenic syndrome: Summary statement. Muscle Nerve. 2001;24:1236-1238.

29. Barkhaus PE. Motor unit number index (MUNIX) and the Chowkidar. Clin Neurophysiol. 2018;129:1714-1715. 